\title{
Retrospective Analysis of the Endovascular and Surgical Treatment Outcomes in Patients with Cerebral Arteriovenous Malformations
}

\author{
Necati UCLER ${ }^{1}$, Tanin OGUR ${ }^{1}$, Ergun DAGLIOGLU ${ }^{2}$ \\ ${ }^{1}$ Adiyaman University, Education and Research Hospital, Neurosurgery Department, Adiyaman, Turkey \\ ${ }^{2}$ Ankara Numune Education and Research Hospital, Neurosurgery Department, Ankara, Turkey \\ Corresponding author: Necati UCLER necati_ucler@yahoo.com
}

\section{ABSTRACT}

AIM: To compare the short-term follow-up outcomes of treatment with liquid embolization agents in 105 patients with low- and medium-grade arteriovenous malformation (AVM) having supra- or infratentorial localization and its reliability and efficacy.

MATERIAL and METHODS: This retrospective study assessed patients diagnosed with grade I, II, III, IV, and V infratentorial AVM according to the Spetzler-Martin grading system who received endovascular treatment and underwent surgical resection. The pretreatment, post-treatment, preoperative, postoperative, and follow-up data and the electronic radiograph findings, epicrisis reports, and clinical follow-up reports were recorded and examined.

RESULTS: AVM was detected in 105 patients, who underwent 132 endovascular treatment sessions in total. Of the 105 patients, 47 were fed with a single venous system; 37 with two venous systems; and 21 with three or more venous systems. The mean follow-up duration was 28 months, and no patient presented with re-hemorrhage. Some of the complications associated with embolization were hemiparesis/hemiplegia, hemihypesthesia, hemorrhage and hematoma, vision disorders and alar defects, cranial nerve paralysis, and aphasia/dysphasia. Meanwhile, seven patients died after treatment.

CONCLUSION: AVM treatment generally includes usage of gamma knife, endovascular treatment, surgical resection, or the combination of such treatments. If surgical resection is performed, a complete and absolute outcome can be achieved. However, complete treatment can be rarely achieved with endovascular therapy alone. Higher morbidity and mortality rates were observed in patients with hemorrhage than in those without.

KEYWORDS: Arteriovenous malformation, Endovascular treatment, Surgery, Complication

ABBREVIATIONS: AVM: Arteriovenous malformation, CVM: Cerebrovascular malformations, DSA: Digital subtraction angiography, ECA: External carotid artery, EVOH: Ethylene vinyl alcohol, GCS: Glasgow Coma Scale, PCA: Posterior cerebral artery, CT: Computed tomography

\section{INTRODUCTION}

$\mathrm{C}$ erebrovascular malformations (CVMs) comprise a heterogeneous group of disorders, including vascular malformations observed in the brain, associated with a wide spectrum of behaviors. Some disorders (i.e., capillary malformations) might be asymptomatic and can be detected incidentally during imaging. CVMs, such as arteriovenous malformation (AVM) and cavernous angioma, might cause unexpected bleeding without any prior triggering symptom (14).

AVM is an irregular angiogenesis characterized by the formation of a direct shunt between the artery and vein, and such a 
lesion can be observed in up to $0.05 \%$ of the population. The annual incidence rates of hemorrhage increase in parallel with age, localization in the deep brain, and deep venous drainage. The annual risk of hemorrhage varies is $1 \%-35 \%$ in patients meeting all these three criteria.

The treatment options are transluminal embolization, surgery, stereotaxic radiosurgery, and the combination of such treatments (multimodal surgery), and the best option is determined based on the patient condition $(4,5)$.

Complete embolization of a lesion is directly associated with the lesion size, and lesions may have one or two feeders of a diameter $<3 \mathrm{~cm}$. In a patient who will undergo surgery, embolization of the lesion before the procedure decreases the risk of surgery and hemorrhage. Moreover, if there is no pressure effect on the lesions with total or gross total embolization, surgery may not be required (5).

Thus, in the present study, we aimed to examine the shortterm follow-up outcomes of 105 patients who have low- or medium-grade AVM with supra- or infratentorial localization and have been treated with liquid embolization agents in 2011-2016. Moreover, the reliability and efficacy of the treatment was assessed.

\section{- MATERIAL and METHODS}

This retrospective study evaluated patients who were diagnosed with grade I, II, III, IV, and V infratentorial AVM according to the Spetzler-Martin grading system and received endovascular treatment and underwent surgical resection. The pre-treatment, post-treatment, preoperative, postoperative, and follow-up data and the electronic radiograph findings, epicrisis reports, and clinical follow-up reports were recorded and examined.

\section{Patient Information}

In the present study, patients with AVM who presented with headache, seizure, and hemorrhage and those incidentally diagnosed with cerebral AVM between 2011 and 2016 were included. No age or gender exclusion criterion was applied.

The patients presented with symptomatic and asymptomatic AVM with supratentorial or infratentorial localization. We conducted treatment with liquid embolization agent + surgical resection via the endovascular path in the interventional radiology unit.

In all patients, prior to treatment, the digital subtraction angiography (DSA) method was used for anterior, posterior cerebral circulation and bilateral external carotid artery (ECA) imaging, and the 3D angiographs of the AVM were obtained for the measurement of the nidus. The other treatment alternatives were discussed with the neurosurgery and radiology departments before the treatment.

\section{Treatment Information and Informed Consent}

The patients who were candidates for endovascular treatment and their relatives were informed about the natural course of AVM as well as the advantages, disadvantages, benefits, and complications of endovascular and surgical treatments. Then, a written informed consent was obtained.

The fundamental clinical examination results and the platelet count, serum creatinine level, and hemoglobin values of the patients were examined prior to the procedure.

\section{Medications}

All patients underwent the treatment procedure under general anesthesia. The medications suitable for general anesthesia were used by the anesthetist team in accordance with the standard protocols of the hospital. In all patients, the bolus dosage was intravenously applied to ensure 2-2.5 folds of the basal-bolus heparin international normalized ratio of the minimum and maximum units at 3000 and 6000 , respectively. The hourly treatment dose was 500-1000 units. At the end of the procedure, the use of heparin was reversed to prevent hemorrhage.

\section{Equipment}

The transfemoral path was used in all patients. Cerebral arteriogram was first conducted. Then, based on the localization of AVM, the guide sheath and catheter were placed into the carotid or vertebral artery. Then, with the use of the micro-catheters, the AVM feeder was catheterized. In relation to the other feeders, the catheterization was performed based on the passage feeder condition. A suitable wire was used to better visualize the flow-directed micro-catheters and prevent the formation of a gap due to a sudden stop. After successfully completing the catheterization, the micro-catheters were rinsed with SF, and based on the gaps inside, their interiors were filled using dimethyl sulfoxide. Then, the injection of ethylene vinyl alcohol (EVOH) was initiated, which was applied slowly and periodically via manual injection for a duration of 20-40 min. The proximal n-butyl cyanoacrylate was applied to prevent reflux in the large feeders of large aneurysms.

The control angiographs were obtained right after completing the procedure, and the evaluations were made based on the AVM obliteration levels and possible complications, such as parent artery occlusion, vasospasm, thromboembolism, EVOH reflux, and recurrent hemorrhage. Unenhanced computed tomography (CT) imaging was performed within the first $3 \mathrm{~h}$ after the procedure to assess for hemorrhage.

\section{RESULTS}

AVM was detected in 105 patients, who underwent a total of 132 endovascular treatment sessions. Subarachnoid hemorrhage and subdural and/or parenchymal hematoma were observed in 32 of 105 patients with AVM. The patients were aged between 11 and 69 years, with a mean age of 37 years. In addition, minor symptoms, such as severe seizure and headache, were observed, and 23 patients had neurological deficits and low Glasgow Coma Scale (GCS) scores during admission. Twentyseven patients had AVM with non-cortical deep localization. Eleven patients presented with infratentorial localization. In 11 patients, cerebral aneurysm was observed on the feeder veins or another localization. Twenty-two patients had AVM with occipital localization; 15 with parietal localization; 11 
with frontal localization; 10 with temporal localization; 15 with tentorial localization; seven with intraventricular localization; six with Sylvian localization; four with frontobasal localization; three with interhemispheric localization; and one with orbital localization. Using the Spetzler- Martin grading system, six patients presented with grade I AVM; 33 with grade II AVM; 29 with grade 3 AVM; 18 with grade 4 AVM; 8 with grade 5 AVM; and 11 with infratentorial AMV. Moreover, 47 patients were fed with a single venous system; 37 with two venous systems; and 21 with three or more venous systems.

In 60 patients, surgical resection was performed during embolization. Total resection was achieved during surgery in all cases. The postoperative residual filling was followed only in three cases. Two patients underwent re-surgery, and reembolization was successfully achieved in one case. Three patients underwent embolization after gamma knife; in 19 patients, gamma knife was recommended as a complementary treatment after endovascular treatment. The mean follow-up duration was 28 months, and none of the patients presented with re-hemorrhage during the follow-up.
The major complications observed in the cases were divided into two groups: secondary to embolization and secondary to surgery. Patients with neurological deficit or low GCS score before embolization or surgery were excluded. However, notably, a significant proportion of patients in this group developed complications (Table I).

The complications associated with embolization were hemiparesis/hemiplegia, hemihypesthesia, hemorrhage and hematoma, vision field defects, cranial nerve paralysis, and aphasia/dysphasia (Tables II, III). Hemorrhage was observed during 6 of 126 sessions of endovascular AVM embolization, and all patients immediately underwent surgery. Despite such treatment, three of these patients died. These cases were considered as mortality associated with endovascular treatment. In three hemorrhagic cases, complete resection could have been achieved during post-embolization AVM surgery; except for one patient, two patients were discharged without any major sequela. Of 105 patients, 8 developed major complications, such as hemiplegia, cerebellar ataxia, and aphasia, and five patients had minor complications, including

Table I: Complications Observed in 13 Patients with AVM according to Type, Presentation, Localization, Feeder, Postop Complications, and Grade

\begin{tabular}{|c|c|c|c|c|c|c|}
\hline No. & Type & Presentation & Localization & Feeder & $\begin{array}{l}\text { Postoperative } \\
\text { complications }\end{array}$ & Grade \\
\hline 1 & 30 & Headache & $\begin{array}{l}\text { Left occipitotentorial } \\
\text { AVM }\end{array}$ & $\begin{array}{l}\text { Left MCA distal } \\
\text { branches }\end{array}$ & $\begin{array}{l}\text { Postoperative visual field } \\
\text { defect }\end{array}$ & 2 \\
\hline 2 & 34 & Headache & $\begin{array}{l}\text { Right occipitotentorial } \\
\text { AVM }\end{array}$ & $\begin{array}{c}\text { Right MCA inferior trunk } \\
+ \text { PCA }\end{array}$ & Visual field defect & 4 \\
\hline 3 & 42 & Headache & Left occipital AVM & Left PCA & Visual field defect & 3 \\
\hline 4 & 30 & Headache & Left occipital AVM & AICA, Left SSA, PICA & $\begin{array}{c}\text { Postoperative hemorrhage, } \\
\text { GCS score of } 6\end{array}$ & 3 \\
\hline 5 & 43 & Headache & $\begin{array}{l}\text { Left frontal } \\
\text { interhemispheric AVM }\end{array}$ & Left MCA & Aphasia & 3 \\
\hline 6 & 19 & SAH Headache & Right occipital AVM & Right MCA, PCA, ECA & Visual field defect & 4 \\
\hline 7 & 24 & $\begin{array}{l}\text { Treatment after the } \\
\text { gamma knife }\end{array}$ & Left cerebellar AVM & Left PCA, PICA, AICA & Nistagmus, ataxia & 3 \\
\hline 8 & 37 & Embolized AVM & $\begin{array}{l}\text { Residual left occipital } \\
\text { AVM }\end{array}$ & Left MCA, PCA & Visual field defect, aphasia & 3 \\
\hline 9 & 21 & $\begin{array}{l}\text { Loss of consciousness, } \\
\text { parietal hematoma }\end{array}$ & $\begin{array}{l}\text { Right occipitotentorial } \\
\text { AVM }\end{array}$ & Right PCA & Paralysis & 4 \\
\hline 10 & 39 & Headache & Left frontal AVM & Left MCA & Paresis & 2 \\
\hline 11 & 38 & $\begin{array}{l}\text { SAK Intraventricular } \\
\text { hemorrhage }\end{array}$ & Left ventricular AVM & Left MCA, choroidal & Paralysis, aphasia, seizure & 3 \\
\hline 12 & 25 & Headache & Right parietal AVM & Right MCA, ACA & Paresis & 3 \\
\hline 13 & 48 & Headache & Left temporal AVM & Left MCA, PCA & Aphasia & 5 \\
\hline
\end{tabular}

MCA: Middle cerebral artery, AVM: Arteriovenous malformation, PCA: Posterior cerebral artery, ECA: External carotid artery, AICA: Anterior Inferior Cerebellar Artery, PICA: Posterior Inferior Cerebellar Artery. 
visual field defect, nystagmus, and hemihypesthesia. Five of the eight patients had major complications with a Modified Rankin Scale score of $\geq 3$.

During treatment, the complications associated with endovascular therapy was observed in three patients (Table

Table II: Morbidity and Mortality Rates according to the Ratio of Embolization

\begin{tabular}{lccc}
\hline Group & Total & Morbidity & Mortality \\
\hline $100-91 \%$ & 34 & 6 & 1 \\
\hline $90-76 \%$ & 18 & 3 & 1 \\
\hline$<75 \%$ & 8 & 2 & 5 \\
\hline
\end{tabular}

Table III: The Complication Rates of AVM Cases: by the SpetzlerMartin Grade

\begin{tabular}{lcc}
\hline Grade & Complication (total) & $\%$ \\
\hline 2 & $2(33)$ & 6 \\
\hline 3 & $6(29)$ & 20 \\
\hline 4 & $3(18)$ & 16 \\
\hline 5 & $1(8)$ & 12 \\
\hline Infratentorial & $1(11)$ & 9 \\
\hline
\end{tabular}

Table IV: Morbidity and Mortality Analysis of AVM Cases by the Feeder Vascular Branch Group: i.e. MCA or ACA or PCA: SingleBranch Group

\begin{tabular}{lccc}
\hline Feeder & Total & Morbidity & Mortality \\
\hline Single system & 47 & 5 & 4 \\
\hline Two systems & 37 & 5 & 3 \\
\hline Multiple feeders & 21 & 5 & 0 \\
\hline
\end{tabular}

IV). Reflux to the left A2 was observed in one case because the endovascular filling material could not be observed, and an immediate reflux to the posterior choroidal artery was found in one patient after the embolization of the posterior cerebral artery (PCA) feeder. In two cases, the reflux occurred because the catheter was removed using a snare since the detachable catheters did not break.

A total of seven patients died (Tables V, VI). Hemorrhage was observed in five cases, whereas the GCS scores of three patients were lower than 14. Three patients died due to complications that developed after endovascular treatment, one patient due to re-hemorrhage during hospital followup, and three patients due to complications that developed after surgery. Two of these cases involved infratentorial AMV, whereas the mean Spetzler-Martin grade of the remaining five patients was 3 . One of these patients had cerebellar localization, one with $4^{\text {th }}$ ventricle localization, two with parietal localization, two with Sylvian location, and one with orbital localization. The mortality rate was high particularly in patients with hemorrhage.

\section{DISCUSSION}

Cerebral AVMs are lesions that are extremely challenging to treat. The radiological anatomy plays an important role in the treatment of such lesions. For both endovascular and surgical resections, knowledge about the anatomy increases the rate of successful surgeries and decreases the risk of complications. Another important point is the difference in the normal vascular structures arising from the AVM feeder. During surgical and endovascular treatments, these normal structures arising from the feeders should be protected and not undergo embolization.

The diameter of the AVM nidus is also an important parameter for successful surgical and endovascular interventions. The success rates, particularly in AVM cases with $\leq 3-\mathrm{cm}$ diameter, significantly differ in the literature (8). 3D DSA is more sensitive in measuring the diameter of the AVM nidus than magnetic resonance imaging angiography or CT angiography (21).

Table V: The Features of Deceased 7 AVM Patients

\begin{tabular}{|c|c|c|c|c|c|c|c|}
\hline Patient & Age & Sex & Presentation & Localization & Feeder & Excision & Grade \\
\hline 1 & 63 & M & $\begin{array}{c}\text { Hemiparesis, left parietal } \\
\text { hematoma }\end{array}$ & Left occipitoparietal AVM & Left ACA PCA & - & 3 \\
\hline 2 & 39 & M & Headache, visual field deficit & Right Sylvian AVM & Right MCA & + & 3 \\
\hline 3 & 25 & M & Headache & Right Sylvian AVM & Right MCA & + & 4 \\
\hline 4 & 52 & M & $\begin{array}{l}\text { Ptosis, headache, hemorrhage, } \\
\text { GCS } 13\end{array}$ & Left orbital AVM & Right ophthalmic AVM & + & 2 \\
\hline 5 & 55 & M & Visual field defect, imbalance & AVM in $4^{\text {th }}$ ventricle & AICA, SSA & + & - \\
\hline 6 & 56 & M & SAH, GCS 8 & $\begin{array}{c}\text { Left cerebellar AVM fed by } \\
\text { artery AVM }\end{array}$ & Left AICA, SSA & + & - \\
\hline 7 & 69 & M & $\begin{array}{l}\text { Previous parenchymal } \\
\text { hemorrhage, headache }\end{array}$ & Left frontotemporal AVM & Right MCA & + & 2 \\
\hline
\end{tabular}


Table VI: Morbidity Rates of AVM Patients by the Spetzler-Martin Grade

\begin{tabular}{lcc}
\hline Grade & Deceased (total) & $\%$ \\
\hline 1 & $0(6)$ & 0 \\
\hline 2 & $2(33)$ & 6 \\
\hline 3 & $2(29)$ & 7 \\
\hline 5 & $1(18)$ & 5 \\
\hline Infratentorial & $0(8)$ & 0 \\
\hline
\end{tabular}

The Spetzler-Martin grading system is a widely used method. However, the Spetzler-Ponce and Lawton grading has replaced the use of such system in recent years $(9,19)$. The evaluation of clinical success and mortality is extremely important particularly after surgical resection $(3,11,12,15,23)$. The clinical outcomes significantly differ in hemorrhagic cases $(1,2)$. The mortality rate was $1 \%$ during the endovascular embolization of cerebral AVM. The hemorrhagic complications might be observed in $2 \%-5 \%$ of the cases. The morbidity rate varied between $10 \%$ and $14 \%(6,7,13,16,17,20,22)$.

In addition, anomalies in adjacent cerebral tissues, aneurysms on the feeding artery, and presence of any pre-treatment hemorrhage and/or hematoma are extremely important for the planning and success of treatment. In particular, patients with aneurysms on the feeding artery are at risk of hemorrhage during feeder catheterization; thus, extreme caution must be exercised. Another important point is that the feeding arteries arise from different vascular structures; for example, the risk of hemorrhage during an endovascular or surgical intervention is high in patients with AVM who underwent vascularization from the middle cerebral artery (MCA), anterior cerebral artery, PCA, and even ECA branches and have multiple feeders; in comparison, the risk is relatively lower in patients with AVM who were fed with a single venous system (particularly the MCA branches). Among the patients with AVM who underwent surgery in our clinic, the mortality and morbidity rates were lower in those fed with a single pedicle than in those fed with two or more feeders.

The relationship between the ectasias observed in the venous system and venous sinuses plays an important role particularly in surgical planning owing to the use of flux only in the venous system and risk of reflux during the endovascular treatment. The presence of veins before the surgical corridor is a significant challenge in planning any AVM surgery, and it should be projected at the planning phase. Premature occlusion of the venous system during embolization increases the risk of preoperative hemorrhage. Similarly, clipping the veins during an AVM resection by considering them as arteries may cause aneurysm or hemorrhage in the AVM nidus. Under any condition, one must pay attention in preventing the development of reflux in these veins during endovascular treatment, and all the feeders must be closed without occlusion. During surgical interventions, the final grade of the nidus feeder resection is to clip the feeding veins and to rapidly excise the AVM.

The success of using endovascular embolization agents may be directly associated with the morphological anatomy. In particular, low- and high-density EVOH polymers are widely used, and it is extremely important to properly use these agents. If embolization is initiated at the point of the feeding artery adjacent to the vein, selecting a high-density embolization agent would decrease the risk of reflux. Conversely, for points far from the vein, selection of a low-density agent might result in a more diffuse and successful embolization with better propagation. Similarly, successful embolization can be achieved using high-density embolization agents in cases of high-flux AVM, and the risk of reflux might be prevented almost completely with the use of low-density agents.

In AVM cases, recurrence is widely observed in patients treated using only the endovascular method, even if radical surgery is successful. It is extremely difficult to recommend embolization as a primary treatment method. It is more appropriate to consider such a method auxiliary to surgery in AVM cases, particularly in the resectable areas.

For cerebral AVMs, embolization during surgery is extremely useful in localizing the lesion because the embolization agent is black, which is why it can be distinguished from the brain tissue; also, it limits the requirement of using neuronavigation. In surgeries performed by assuming that the adjacent cerebral vascular structures are protected, the gross excision of the vascular structures that are full of embolization agent indicates the excision of the nidus. In relation to this, the exception is maybe to partially leave the nidus as residual particularly in diffuse AVMs. The complexity of distinguishing the artery from the vein during surgery can be eliminated. In addition, particularly for cases with an embolization rate of $95 \%$ or higher, the risk of hemorrhage during EVM excision is almost completely eliminated, and the requirement of using Doppler during surgery is partially overcome. In other words, excision may be easier to achieve in such case than in an avascular meningioma case.

The success rate decreases in multi-feeder AVM cases and increases in single-feeder AVMs. One of the reasons is that endovascular treatment is assumed to be difficult since the multi-feeder AVMs are generally large and these types of AVMs generally have complex vascular structures. In cases in which complete surgical resection is challenging, the use of endovascular treatment or the combination of endovascular treatment and gamma knife radiosurgery can be an option.

In addition, if AVM treatment has been initiated using any method, then the treatment must be completed. In the A Randomized Trial of Unruptured Brain Arteriovenous Malformations study, the risk of hemorrhage increases if the procedure is not completed (10). If surgical resection is not considered for treatment, either embolization or the gamma knife method or the combination of both can be the other options, which is based on what method suits the condition of the patient. 
Distinguishing hemorrhagic from non-hemorrhagic AVM is exremely important for the selection of treatment parameters. In particular, for hemorrhagic cases, both high risk for preoperative re-hemorrhage and the advantage of easily performing dissection in hemorrhagic cases validate the need for surgical intervention. Considering the findings of the present study showing that four of six patients who presented with hemorrhage prior to surgery have been admitted due to re-hemorrhage, those who had hemorrhage prior to surgery are at higher risk for re-hemorrhage.

Patients with infratentorial AVM should be examined separately from those with supratentorial AMV. Thus, the Spetzler-Martin grading was not applied to these patients in our series. Young patients with infratentorial AVM are at high risk of hemorrhage (5-6 times higher than in supratentorial cases), and this result indicates that the treatment of these patients must be immediately started (18). Hemorrhage was observed in the second session after the first embolization session of a patient with cerebellar AVM who was admitted to our clinic due to hemorrhage. In such cases, caution must be observed. Moreover, patients must be closely monitored for hydrocephaly due to the posterior fossa localization. Watchful waiting or even prophylactic ventriculostomy might be preferred for patients who underwent embolization. In our clinical series, the patient with AVM in the $4^{\text {th }}$ ventricle (Patient 80) died despite cautious monitoring after endovascular treatment because of cardiac arrest due to hydrocephaly. Although ventriculostomy was immediately performed, the clinical condition of the patient did not change.

An interesting relationship was observed between the embolization rate and surgical success and risk in AVM cases. In our series, the embolization rates in patients with AVM who underwent endovascular obliteration or surgical resection after the session(s) were divided into three groups: $\leq 75 \%$, between 75 and $90 \%$, and $\geq 90 \%$. Eight patients presented with an embolization rate of $75 \%$ or lower; 18 with an embolization rate between 75 and $90 \%$; and 34 with an embolization rate of $90 \%$ or higher. The complication rates of the groups are presented in Table II.

\section{CONCLUSION}

The treatment of AVM is extremely complex, and the treatment options are generally gamma knife, endovascular treatment, surgical resection, or the combination of such treatments. In particular, when surgical resection can be performed, an absolute and complete outcome can be achieved. By contrast, complete treatment with endovascular therapy alone can be rarely achieved. Endovascular treatment decreases the risks associated with surgical resection. However, such procedure results in higher mortality and morbidity rates in hemorrhagic than in non-hemorrhagic patients according to the SpetzlerMartin grading system.

\section{- DISCLOSURE}

The authors report no conflict of interest concerning the materials or methods used in this study or the findings specified in this paper.

\section{REFERENCES}

1. Appelboom G, Hwang BY, Bruce SS, Piazza MA, Kellner $\mathrm{CP}$, Meyers PM, Connolly ES: Predicting outcome after arteriovenous malformation associated intracerebral hemorrhage with the original $\mathrm{ICH}$ score. World Neurosurg 78:646-650, 2012

2. Bir SC, Maiti TK, Konar S, Nanda A: Overall outcomes following early interventions for intracranial arteriovenous malformations with hematomas. J Clin Neurosci 23:95-100, 2016

3. Bradac O, Charvat F, Benes V: Treatment for brain arteriovenous malformation in the 1998-2011 period and review of the literature. Acta Neurochir (Wien) 155:199-209, 2013

4. Colby GP, Coon AL, Huang J, Tamargo RJ: Historical perspective of treatments of cranial arteriovenous malformations and duralarteriovenous fistulas. Neurosurg Clin N Am 23:15-25, 2012

5. Deshaies EM, Eddleman CS, Boulos AS: Handbook of Neuroendovascular Surgery. Thieme Medical Publishers, 2012

6. Elsenousi A, Aletich VA, Alaraj A: Neurological outcomes and cure rates of embolization of brain arteriovenous malformations with n-butyl cyanoacrylate or Onyx: A metaanalysis. J Neurointerv Surg 8:265-272, 2016

7. Hartmann A, Stapf C, Hofmeister C, Mohr JP, Sciacca RR, Stein BM, Faulstich A, Mast $\mathrm{H}$ : Determinants of neurological outcome after surgery for brain arteriovenous malformation. Stroke 31:2361-2364, 2000

8. Jeon HJ, Park KY, Kim SY, Lee JW, Huh SK, Lee KC: Surgical outcomes after classifying Grade III arteriovenous malformations according to Lawton's modified SpetzlerMartin grading system. Clin Neurol Neurosurg 124:72-80, 2014

9. Lawton MT, Kim H, McCulloch CE, Mikhak B, Young WL: A supplementary grading scale for selecting patients with brain arteriovenous malformations for surgery. Neurosurgery 66:702-713, 2010

10. Mohr JP, Parides MK, Stapf C, Moquete E, Moy CS, Overbey JR, Salman RA, Vicaut E, Young WL, Houdart E, Cordonnier C, Stefani MA, Hartmann A, von Kummer R, Biondi A, Berkefeld J, Klijn CJM, Harkness K, Libman R, Barreau X, Moskowitz AJ: Medical management with or without interventional therapy for unruptured brain arteriovenous malformations (ARUBA): A multicentre, non-blinded, randomised trial. Lancet 383:614-621, 2014

11. Morgan MK, Rochford AM, Tsahtsarlis A, Little N, Faulder KC: Surgical risks associated with the management of Grade I and II brain arteriovenous malformations. Neurosurgery 54: 832-837, 2004; discussion 837-839

12. Nair AP, Kumar R, Mehrotra A, Srivastava AK, Sahu RN, Nair P: Clinical, radiological profile and outcome in pediatric Spetzler-Martin grades I-III arteriovenousmal formations. Childs Nerv Syst 28:593-598, 2012

13. n-BCA Trail Investigators: $\mathrm{N}$-butyl cyanoacrylate embolization of cerebral arteriovenous malformations: Results of a prospective, randomized, multi-center trial. AJNR Am J Neuroradiol 23:748-755, 2002 
14. Osborn AG: Osborn's brain: Imaging, pathology, and anatomy. Lippincott Williams \& Wilkins, 2012

15. Pandey P, Marks MP, Harraher CD, Westbroek EM, Chang SD, Do HM, Levy RP, Dodd RL, Steinberg GK: Multimodality management of Spetzler-Martin Grade III arteriovenous malformations. J Neurosurg 116:1279-1288, 2012

16. Pierot L, Kadziolka K, Litré F, Rousseaux P: Combined treatment of brain AVMs with use of Onyx embolization followed by radiosurgery. AJNR Am J Neuroradiol 34:13951400, 2013

17. Potts MB, Zumofen DW, Raz E, Nelson PK, Riina HA: Curing arteriovenous malformations using embolization. Neurosurg Focus 37:E19, 2014

18. Rodríguez-Hernández A, Kim H, Pourmohamad T, Young WL, Lawton MT; University of California, San Francisco Arteriovenous Malformation Study Project: Cerebellar arteriovenous malformations: Anatomic subtypes, surgical results, and increased predictive accuracy of the supplementary grading system. Neurosurgery 71: 1111-1124, 2012
19. Steiger HJ, Fischer I, Rohn B, Turowski B, Etminan N, Hänggi D: Microsurgical resection of Spetzler-Martin grades 1 and 2 unruptured brain arteriovenous malformations results in lower long-term morbidity and loss of quality-adjusted lifeyears (QALY) than conservative management-results of a single group series. Acta Neurochir (Wien) 157:1279-1287, 2015

20. Westphal M, Cristante L, Grzyska U, Freckmann N, Zanella F, Zeumer H, Herrmann HD: Treatment of cerebral arteriovenous malformations by neuroradiological intervention and surgical resection. Acta Neurochir (Wien) 130:20-27, 1994

21. Wu C, Ansari SA, Honarmand AR, Vakil P, Hurley MC, Bendok BR, Carr J, Carroll TJ, Markl M: Evaluation of 4D vascular flow and tissue perfusion in cerebral arteriovenous malformations: Influence of Spetzler-Martin grade, clinical presentation, and AVM risk factors. AJNR Am J Neuroradiol 36:1142-1149, 2015

22. Xu F, Ni W, Liao Y, Gu Y, Xu B, Leng B, Song D: Onyx embolization for the treatment of brain arteriovenous malformations. Acta Neurochir (Wien) 153:869-878, 2011

23. Zhao J, Yu T, Wang S, Zhao Y, Yang WY: Surgical treatment of giant intracranial arteriovenous malformations. Neurosurgery 67:1359-1370, 2010 